\title{
Chemical composition of semi-regular variable giants ${ }^{\star}$
}

\author{
S. M. Andrievsky ${ }^{1,2,3}$, S. A. Korotin ${ }^{2,3}$, and P. Martin ${ }^{4}$ \\ 1 GEPI, Observatoire de Paris-Meudon, 92125 Meudon Cedex, France \\ 2 Department of Astronomy and Astronomical Observatory, Odessa National University, T.G. Shevchenko Park, 65014 Odessa, \\ Ukraine \\ ${ }^{3}$ Isaac Newton Institute of Chile, Odessa Branch, Ukraine \\ e-mail: scan@deneb1.odessa.ua; serkor@skyline.od.ua \\ 4 Canada-France-Hawaii Telescope, PO Box 65-1238, Mamalahoa Hwy, HI 96743, USA \\ e-mail: martin@cfht.hawaii.edu
}

Received 14 August 2006 / Accepted 4 December 2006

\begin{abstract}
Aims. The aim of this work was to derive chemical abundances in 5 stars that were previously reported to be SRd variable stars from the halo population, and, in particular, to check if manganese does show significant underabundance in our program stars similar to that of TY Vir, first detected by Luck \& Bond.

Methods. Elemental abundances have been found through the LTE and NLTE analyses, applied to high-resolution $(R \sim 50000)$ spectra.

Results. We have derived the abundances of 18 chemical elements in RX Vir, AB Leo, CK Vir, SV UMa, and TY Vir, and made the following conclusions.

Conclusions. RX Vir is a typical solar-like star. Its classification as a halo population SRd variable star should be ruled out. The remaining four stars of our program have chemical composition and fundamental parameters that confirm their status as metaldeficient halo giants. All studied SRd giants have relative-to-solar elemental abundances typical of the field halo stars of metallicity -1.8 . There is only one exception, which is the relative manganese abundance. All SRd program stars show uniform [Mn/Fe] ratios that are typical of the stars with metallicity $[\mathrm{Fe} / \mathrm{H}] \approx-3.5$. The reason for such a low manganese abundance in these stars is unknown.
\end{abstract}

Key words. stars: variables: general - stars: abundances

\section{Introduction}

According to the recent version of the General Catalogue of Variable Stars (http://www.sai.msu.su/gcvs/gcvs/), the group of semi-regular variable giants and supergiants (SRd) comprises about 220 stars of F, G, K (and M) spectral classes (including those which are suspected SRd variables). Photometrical amplitudes of their variability in some cases may achieve 4 magnitudes (but usually are less than 1-2 mag.), while periods extend from one month to about 3 years. From their kinematical properties these stars are supposed to belong to the Galactic halo population.

There are only a few papers in the literature discussing the chemical composition determined spectroscopically for these stars. Among the early studies, Preston \& Wallerstein (1963) have analysed two stars SX Her and TY Vir, and found that they are metal-deficient, compared to the Sun (later these two stars and additionally CK Vir were analysed by Leep \& Wallerstein 1981). More recently, Luck \& Bond (1985) investigated the metal-poor star TY Vir, and Andrievsky et al. (1985) used high-resolution spectroscopy to study the SRd giant candidate, VW Dra, and found it to be a metal-deficient star.

* Based on observations obtained at the Canada-France-Hawaii Telescope (CFHT), which is operated by the National Research Council of Canada, the Institute National des Sciences de l'Univers of the Centre National de la Recherche Scientifique of France, and the University of Hawaii.
Recently, Giridhar et al. (1998, 1999, 2000) published a series of interesting papers especially devoted to the abundance determination of SRd type stars. Those authors investigated 12 stars that are classified as semi-regular variables. They found that some stars in this group have to be considered as misclassified SRds, while the following stars: AB Leo, SV UMa, WY And, VW Eri, UW Lib, KK Aql, AG Aur, Z Aur, W LMi, and WW Tau appeared to be really metal-poor halo giants with chemical peculiarities identical to the halo dwarfs, subgiants, and unevolved giants of the same metallicity. In those papers Giridhar et al. (1998, 1999, 2000) also raised some questions concerning the typical chemical composition of SRd stars, and, in particular, about its connection to the evolutionary status of these stars. Unfortunately, the data on carbon and nitrogen abundances were not available to these authors for all the stars they investigated. That made it more complicated in some cases to conclude whether investigated SRd variables are the tip-RGB stars, or AGB stars, and whether they can be considered as preRV Tau stars. The authors of this series of papers believe that SRd stars are evolutionary connected with RV Tau stars. There is one particular peculiarity detected by Luck \& Bond (1985) in TY Vir: a strong deficiency of manganese $(\mathrm{Mn})$ in this star. Surprisingly, for some other SRd stars, Giridhar et al. (1998, 1999, 2000) did not report about any significant Mn overdeficiency. The main objective of the work presented here was to derive abundances in 5 stars that were previously reported to be the halo population SRd variable stars, and, in particular, to check if 
Table 1. Characteristics of program stars.

\begin{tabular}{cccccc}
\hline \hline Star & $V$ & $T_{\text {eff }}, \mathrm{K}$ & $\log g$ & $V_{\mathrm{t}}, \mathrm{km} \mathrm{s}^{-1}$ & Rem \\
\hline RX Vir & $8.7-9.1$ & 5950 & 4.00 & 1.5 & SRd: \\
AB Leo & $10.7-13.2$ & 4130 & 0.00 & 2.4 & SRd \\
CK Vir & $10.2-11.0$ & 4200 & 0.20 & 2.3 & SRd \\
SV UMa & $9.1-10.6$ & 4300 & 0.00 & 2.4 & SRd \\
TY Vir & $8.0-8.5$ & 4350 & 0.30 & 2.4 & SRd \\
\hline
\end{tabular}

Classification of the stars is given following the GCVS. According to the $A S A S$ database (http://www.astrouw.edu.pl/ gp/asas/ asas.html) RX Vir seems to be a constant star.

Mn does show significant underabundance in our program stars similar to that in TY Vir first detected by Luck \& Bond (1985).

\section{Observations}

We selected several SRd stars for our observation campaign using the Canada-France-Hawaii $3.6 \mathrm{~m}$ telescope. Observations were carried out during photometric nights in 2003 with the fiber-fed Gecko spectrograph equipped with a EEV1 $2048 \times$ $4500 \mathrm{CCD}$ camera (binned $2 \times 2$ ). The resolving power provided by this combination was about 50000 .

The list of the stars and some of their characteristics are given in Table 1. For each star of our program, 6 spectral fragments (5 for TY Vir) centered at 6040, 6130, 6220, 6310, 6400, and $6490 \AA$ were observed. Each spectral segment is $80 \AA$; exposures times were adjusted in function of the star magnitude and vary from $930 \mathrm{~s}$ to $1800 \mathrm{~s}$ per fragment. For all the stars the $\mathrm{S} / \mathrm{N}$ ratio within the observed fragments is in the interval $100-150$. The spectra were extracted from the raw frames using standard IRAF procedures. Additional work with the spectra (continuum placement, equivalent width measurements, etc) was carried out using the DECH20 code (Galazutdinov 1992).

\section{Analysis}

Equivalent widths of the lines selected were measured using a Gaussian approximation. For all the spectra of the stars in our sample, the absorption lines look narrow and symmetric (except for SV UMa where the lines are symmetric but rather broad). This enabled us to measure the line equivalent widths with a high accuracy.

The list of the unblended lines has been selected from the VALD database (http://ams.astro.univie.ac.at/ vald/). For all those lines this database lists the excitation potentials, oscillator strengths, and broadening parameters. Our spectroscopic analysis was based on LTE Kurucz's atmosphere models (Kurucz 1992). For all of the chemical elements investigated (except for oxygen, sodium, and magnesium) the elemental abundances were derived with the help of Kurucz's WIDTH9 code. Abundances of $\mathrm{O}, \mathrm{Na}$, and $\mathrm{Mg}$ were found using the NLTE spectral synthesis based on the modified version of MULTI code (see Carlsson 1986, for the MULTI description; and Korotin et al. 1999a; Korotin \& Mishenina 1999; Andrievsky et al. 2001, and references therein, for the description of code modification and applied atomic models).

The effective temperature $T_{\text {eff }}$ for each program star was estimated using the line depth ratios. The method based on the sensitive temperature indicators was developed for giant stars by Kovtyukh et al. (2006). The method is independent of the interstellar reddening, and only marginally dependent on individual
Table 2. Elemental abundances in RX Vir.

\begin{tabular}{|c|c|c|c|}
\hline Ion & {$[\mathrm{El} / \mathrm{H}]$} & $\sigma$ & NL \\
\hline C I & +0.00 & - & 1 \\
\hline O I & +0.18 & - & 1 \\
\hline $\mathrm{Na} I$ & +0.10 & 0.11 & 2 \\
\hline $\operatorname{Mg~I}$ & +0.14 & 0.10 & 1 \\
\hline Si I & +0.15 & 0.08 & 13 \\
\hline Si II & -0.04 & 0.01 & 2 \\
\hline $\mathrm{Ca} \mathrm{I}$ & +0.09 & 0.12 & 11 \\
\hline Sc I & +0.34 & 0.16 & 2 \\
\hline Sc II & +0.02 & 0.11 & 4 \\
\hline $\mathrm{Ti} \mathrm{I}$ & +0.21 & 0.10 & 8 \\
\hline Ti II & +0.07 & 0.08 & 3 \\
\hline V I & +0.27 & 0.09 & 19 \\
\hline V II & -0.02 & - & 1 \\
\hline Cr I & +0.17 & 0.02 & 1 \\
\hline Cr II & +0.19 & - & 1 \\
\hline Mn I & -0.15 & 0.04 & 3 \\
\hline $\mathrm{Fe} \mathrm{I}$ & +0.07 & 0.13 & 74 \\
\hline $\mathrm{Fe}$ II & +0.00 & 0.10 & 12 \\
\hline Co I & +0.28 & 0.09 & 4 \\
\hline Ni I & +0.07 & 0.10 & 19 \\
\hline $\mathrm{Zr}$ II & -0.02 & - & 1 \\
\hline Ba II & +0.10 & 0.01 & 1 \\
\hline La II & +0.19 & 0.03 & 2 \\
\hline $\mathrm{Ce}$ II & +0.11 & - & 1 \\
\hline Pr II & +0.05 & 0.27 & 2 \\
\hline
\end{tabular}

$\mathrm{NL}$ - the number of lines used in analysis.

characteristics of stars, such as rotation, microturbulence, and metallicity. The mean error in $T_{\text {eff }}$ is no larger than $100-150 \mathrm{~K}$.

As it was showed by Kovtyukh \& Andrievsky (1999), the best and most reliable way to estimate the microturbulent velocity in the supergiant/giant atmosphere (and thus to subsequently determine the gravity value) is to use the Fe II lines. Unfortunately, there are only a few Fe II lines available in each spectrum of our stars; therefore we applied the standard method of the $V_{\mathrm{t}}$ and $\log g$ determination based on the use of Fe I lines. The atmosphere parameters of our program stars are given in Table 1. Since we used the VALD's compilation of oscillator strengths, we have eliminated the possible random errors in the derived abundances by applying the line-by-line comparison of stellar elemental abundances with the solar ones, which have been determined with the same log $g f$ values, solar atmosphere model from the Kurucz's grid, and microturbulent velocity of $1.5 \mathrm{~km} \mathrm{~s}^{-1}$. The results are presented in the next section.

\section{Abundance results}

Tables 2-4 list the relative-to-solar abundances for the stars of our sample. Graphically, the distribution of the chemical elements in the stars studied is shown in Fig. 1. For the stars common to both samples (AB Leo and SV UMa) our abundances only marginally agree with the abundances derived by Giridhar et al. (1998, 1999, 2000) (see Tables 3-4); for some elements (like Mn, for instance) the discrepancy is extremely big. Such a discrepancy is partially caused by the difference in the effective temperature values adopted by us and Giridhar et al. (1998, 1999, 2000). For SV UMa, their value is $4500 \mathrm{~K}$, that is $200 \mathrm{~K}$ higher than ours. It is also difficult to evaluate an accuracy of the abundances reported by those authors, since no individual statistical errors of the derived abundances are given in their paper. At the same time we are in good agreement with Luck \& Bond (1985) on the abundance results for TY Vir. 
Table 3. Elemental abundances in AB Leo and CK Vir.

\begin{tabular}{cccccc|cccc}
\hline \hline \multicolumn{1}{c}{ AB Leo } & \multicolumn{5}{c}{ CK Vir } \\
\hline Ion & {$[\mathrm{El} / \mathrm{H}]$} & $\sigma$ & $\mathrm{NL}$ & $\mathrm{GET} 98$ & {$[\mathrm{El} / \mathrm{Fe}]$} & {$[\mathrm{El} / \mathrm{H}]$} & $\sigma$ & $\mathrm{NL}$ & {$[\mathrm{El} / \mathrm{Fe}]$} \\
\hline O I & -1.22 & - & 1 & -1.0 & +0.74 & -1.10 & - & 1 & +0.78 \\
$\mathrm{Na}$ I & -1.94 & 0.10 & 2 & -1.9 & +0.02 & -2.00 & 0.15 & 2 & -0.12 \\
$\mathrm{Mg}$ I & -1.48 & - & 1 & - & +0.48 & -1.53 & - & 1 & +0.35 \\
Si I & -1.35 & 0.13 & 5 & -1.7 & +0.61 & -1.52 & 0.08 & 6 & +0.36 \\
Ca I & -1.62 & 0.17 & 7 & -1.6 & +0.34 & -1.57 & 0.16 & 7 & +0.31 \\
Sc I & -1.79 & - & 1 & - & +0.17 & -2.35 & 0.06 & 2 & -0.47 \\
Sc II & -1.92 & 0.14 & 2 & -1.2 & +0.04 & -1.79 & 0.06 & 4 & +0.09 \\
Ti I & -1.54 & 0.04 & 6 & - & +0.42 & -1.64 & 0.07 & 5 & +0.24 \\
Ti II & -1.70 & - & 1 & - & +0.26 & -1.67 & 0.09 & 2 & +0.21 \\
V I & -1.75 & 0.08 & 14 & -1.8 & +0.21 & -1.98 & 0.10 & 14 & -0.10 \\
V II & -2.18 & - & 1 & - & -0.22 & - & - & - & - \\
Cr I & -2.18 & 0.13 & 2 & -1.9 & -0.22 & -2.11 & - & 1 & -0.23 \\
Mn I & -2.79 & 0.09 & 3 & - & -0.83 & -2.77 & 0.12 & 3 & -0.89 \\
Fe I & -1.96 & 0.21 & 59 & -1.6 & - & -1.88 & 0.19 & 64 & - \\
Fe II & -1.98 & 0.25 & 5 & -1.6 & - & -1.89 & 0.07 & 5 & - \\
Co I & -1.68 & 0.16 & 5 & - & +0.28 & -1.68 & 0.08 & 5 & +0.20 \\
Ni I & -2.05 & 0.19 & 7 & -1.7 & -0.09 & -2.02 & 0.11 & 11 & -0.14 \\
Zr I & -2.01 & 0.03 & 2 & - & -0.05 & -2.11 & 0.03 & 2 & -0.23 \\
Zr II & - & - & - & - & - & -1.69 & - & 1 & +0.19 \\
Ba II & -2.18 & 0.04 & 2 & -1.3 & -0.22 & -1.77 & 0.08 & 2 & +0.11 \\
La II & -1.95 & - & 1 & - & +0.01 & -2.08 & 0.03 & 2 & -0.20 \\
Ce II & -2.47 & - & 1 & - & -0.51 & -2.10 & - & 1 & -0.22 \\
Pr II & -2.01 & - & - & - & -0.05 & - & - & - & - \\
Nd II & -1.60 & 0.30 & 2 & - & +0.36 & -1.61 & 0.07 & 2 & +0.27 \\
\hline
\end{tabular}

GET98 - [El/H] from Giridhar et al. (1998).

Table 4. Elemental abundances in SV UMa and TY Vir.

\begin{tabular}{cccccc|ccccc}
\hline \hline & \multicolumn{1}{c}{ SV UMa } & \multicolumn{7}{c}{ TY Vir } \\
\hline Ion & {$[\mathrm{El} / \mathrm{H}]$} & $\sigma$ & $\mathrm{NL}$ & $\mathrm{GET} 98$ & {$[\mathrm{El} / \mathrm{Fe}]$} & {$[\mathrm{El} / \mathrm{H}]$} & $\sigma$ & $\mathrm{NL}$ & {$[\mathrm{El} / \mathrm{Fe}]$} & $\mathrm{LB} 85$ \\
\hline O I & -1.00 & - & 1 & - & +0.87 & -1.03 & - & 1 & +0.68 & -0.70 \\
$\mathrm{Na}$ I & - & - & - & - & - & - & - & - & - & -1.7 \\
$\mathrm{Mg}$ I & - & - & - & -1.2 & - & -1.16 & - & 1 & +0.55 & -1.7 \\
$\mathrm{Si}$ I & - & - & - & - & - & -1.14 & 0.16 & 6 & +0.57 & -1.1 \\
$\mathrm{Si} \mathrm{II}$ & -0.77 & - & 1 & - & +1.10 & - & - & - & - & - \\
$\mathrm{Ca}$ I & -1.87 & 0.07 & 4 & -1.6 & +0.00 & -1.54 & 0.12 & 3 & +0.17 & -1.5 \\
Sc I & -1.54 & 0.08 & 2 & - & +0.33 & -2.19 & - & 1 & -0.48 & - \\
Sc II & - & - & - & -1.5 & - & -1.61 & 0.09 & 4 & +0.10 & -1.4 \\
Ti I & -1.51 & 0.02 & 2 & - & +0.36 & -1.47 & 0.15 & 8 & +0.24 & -1.3 \\
Ti II & -1.64 & - & 1 & -1.2 & +0.23 & -1.37 & - & 12 & +0.34 & -1.3 \\
V I & -1.98 & 0.13 & 4 & -0.9 & -0.11 & -1.70 & 0.12 & 12 & +0.01 & -1.6 \\
V II & - & - & - & - & - & -1.55 & - & 1 & +0.16 & - \\
Cr I & - & - & - & -1.7 & - & -1.87 & - & 1 & -0.16 & -1.8 \\
Mn I & -2.93 & 0.02 & 2 & -1.6 & -1.06 & -2.60 & 0.09 & 3 & -0.89 & -2.4 \\
Fe I & -1.87 & 0.17 & 29 & -1.4 & - & -1.71 & 0.19 & 59 & - & -1.4 \\
Fe II & -1.85 & 0.08 & 3 & -1.3 & - & -1.71 & 0.11 & 4 & - & -1.4 \\
Co I & -1.64 & - & 1 & - & +0.23 & -1.35 & 0.06 & 3 & +0.36 & - \\
Ni I & -1.97 & 0.11 & 7 & -1.4 & -0.10 & -1.82 & 0.17 & 8 & -0.11 & -1.7 \\
Zr I & -2.00 & - & 1 & - & -0.13 & - & - & - & - & - \\
Zr II & - & - & - & - & - & - & - & - & - & - \\
Ba II & -1.20 & 0.28 & 2 & - & +0.67 & -1.17 & - & 1 & +0.54 & -1.2 \\
La II & -1.77 & - & 1 & - & +0.10 & -1.60 & 0.15 & 1 & +0.11 & - \\
Ce II & - & - & 1 & - & - & -1.83 & - & 1 & -0.12 & - \\
Nd II & -1.67 & - & 1 & - & +0.20 & -1.39 & 0.12 & 2 & +0.32 & - \\
\hline
\end{tabular}

GET98 - [El/H] from Giridhar et al. (1998); LB85 - [El/H] from Luck \& Bond (1985).

\section{Discussion}

First of all, one has to note that RX Vir is not a halo population star. With its atmosphere parameters it belongs to the solar-like stars, and its chemical composition is common to the thin disc population stars. High surface gravity $(\log g=4.0)$, found in this study, clearly rules out an SRd luminous star and shows instead that the star is a $\mathrm{G}$ dwarf. Moreover, Jones \& Fisher (1984) measured a radial velocity of $+18.5 \mathrm{~km} \mathrm{~s}^{-1}$ for this star. This low velocity is consistent with our present conclusion that the star is a normal disc star.

The variability of RX Vir was noted by Gaposhkin (1952) with period of about 200 days and amplitude 0.4 mag., but later photoelectric observations by Eggen (1973) did not confirm the 


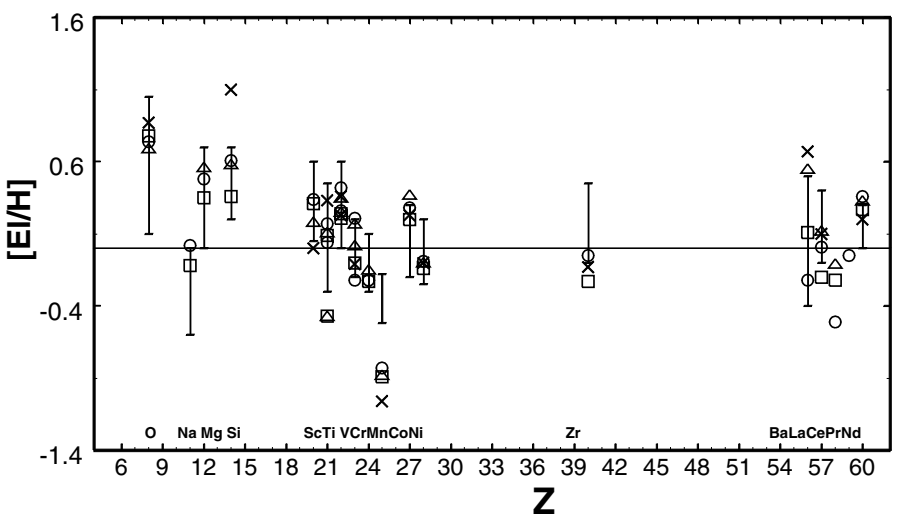

Fig. 1. The distribution of chemical elements in the sampled stars (RX Vir is excluded, see explanation in the text). AB Leo - circles, CK Vir - squares, SV UMa - crosses, TY Vir - triangles. The vertical bars show the approximate abundance variations amongst the halo stars with $[\mathrm{Fe} / \mathrm{H}]=-1.85$.

variability of this star. Thus, the classification of RX Vir as SRd(:) given by the GCVS should be rejected. Other stars of our program have atmosphere parameters and metallicity that are typical for the halo giants.

For discussing the abundances in our SRd stars, we will refer those abundances to the observed $[\mathrm{El} / \mathrm{Fe}]$ values that were reported in several previous studies. For the chemical elements considered here, the approximate limits that comprise the bulk of the halo stars with $[\mathrm{Fe} / \mathrm{H}]=-1.85$ investigated in the literature (this value corresponds to the mean $[\mathrm{Fe} / \mathrm{H}]$ for our four program SRds) have been taken from compilations of Samland (1998) and Goswami \& Prantzos (2000). These data were supplemented/improved using the relative abundances published in more recent papers by Fulbright (2000), Stephens \& Boesgaard (2002), Fulbright (2002), Johnson (2002), Takeda et al. (2003), Gratton et al. (2003), Simmerer et al. (2004), and Zhang \& Zhao (2005). Some of these sources also provide the necessary ratios for the heavy elements (except for cerium and praseodymium). The limits found are showed in Fig. 1.

For the most studied elements in this paper, there are no significant deviations of abundances in our SRd stars from what is expected for metal-deficient halo stars of a similar metallicity. Nevertheless, there are two exceptions: a signicant depletion of $\mathrm{Mn}$ in all program giant stars, and an increased silicon abundance in SV UMa. The latter is based on abundance estimates only from one line (6347 $\AA$ ), and apparently requires an independent confirmation with a larger number of lines. Therefore, we consider this finding as a preliminary result. An increased barium abundance is due to the saturation of the barium lines in giants of our program that have equivalent widths larger than $200 \mathrm{~mA}$.

The Mn abundance is obviously very low in our four SRd stars, and at the same time the ratio $[\mathrm{Mn} / \mathrm{Fe}]$ is surprisingly uniform. We did not take into account the hyper-fine splitting of the lines 6013,6016 , and $6021 \AA$ that were used to derive the Mn abundance in program stars (since these lines are quite weak less than $60 \mathrm{~m} \AA$ for all the stars, and thus not expected to be significantly influenced by the splitting), but this means that the corrected value $[\mathrm{Mn} / \mathrm{H}]$ should be even slightly lower. The spectrum synthesis for Mn lines in our program stars is shown in Fig. 2.
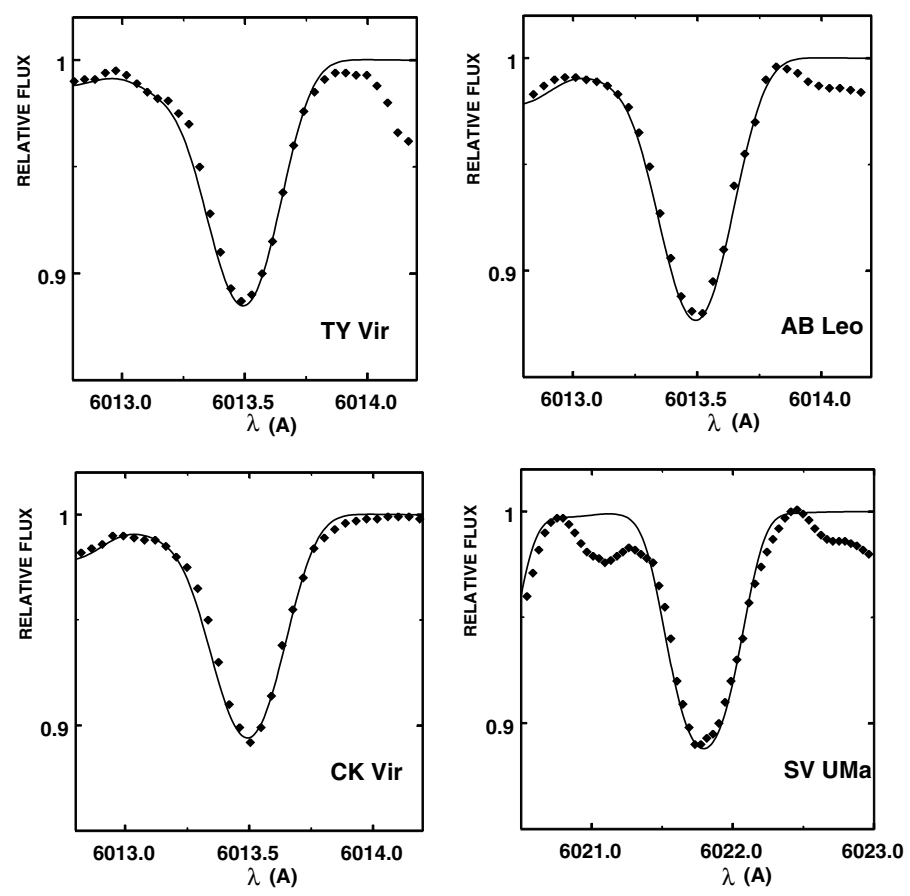

Fig. 2. Mn I line profile fitting.

For the first time, a remarkable Mn deficiency was noted by Luck \& Bond (1985) for TY Vir. The relative abundance of this element $[\mathrm{Mn} / \mathrm{Fe}]$ found in our stars roughly corresponds to what is observed in extremely metal-poor stars of metallicity of about $[\mathrm{Fe} / \mathrm{H}]=-3.5$ (see, e.g., Barklem et al. 2005). No stars with such a low ratio $[\mathrm{Mn} / \mathrm{Fe}]$ are found to be present in the Galactic halo at a metallicity of about $[\mathrm{Fe} / \mathrm{H}]=-2$ (according to the special survey of Mn abundance in cluster and field stars performed by Sobeck et al. 2006).

\section{Conclusion}

We have investigated 5 stars that were reported to be SRd variable stars from the halo population. Based on the elemental abundances derived for these stars we can draw three main conclusions:

- RX Vir has atmosphere parameters and chemical abundances that are typical for the solar-like star. Its classification as a halo population SRd variable star should be ruled out. Moreover, there appears to be no evidence that RX Vir is variable.

- The remaining four stars of our program all have a chemical composition and fundamental parameters confirming their status as metal-deficient halo giants.

- We showed that all studied SRd giants have relative-to-solar elemental abundances typical of the field halo stars of metallicity -1.8 . There is only one striking exception: the relative $\mathrm{Mn}$ abundance. All SRd program stars show uniform $[\mathrm{Mn} / \mathrm{Fe}]$ ratios that are typical for the stars with metallicity $[\mathrm{Fe} / \mathrm{H}] \approx-3.5$. The reason of such a low $\mathrm{Mn}$ abundance in these stars is unknown.

Acknowledgements. S.M.A. kindly acknowledges the CNRS for financial support and the administration of the Paris-Meudon Observatoire for its hospitality during his stay in Meudon where part of this work was performed. We acknowledge the CFHT Support Staff for their help during the observations. The authors thank the anonymous referee for offering important suggestions. 


\section{References}

Andrievsky, S. M., Makarenko, E. N., \& Fenina, Z. N. 1985, PrKFi, 20, 60 Andrievsky, S. M., Kovtyukh, V. V., Korotin, S. A., Spite, M., \& Spite, F. 2001, A\&A, 367, 605

Andrievsky, S. M., Egorova, I. A., Korotin, S. A., \& Burnage, R. 2002, A\&A, 389,519

Barklem, P. S., Christlieb, N., Beers, T. C., et al. 2005, A\&A, 439, 129

Carlsson, M. 1986, Uppsala Obs. Rep., 33

Eggen, O. J. 1973, PASP, 85, 42

Fulbright, J. P. 2000, AJ, 120, 1841

Fulbright, J. P. 2002, AJ, 123, 404

Galazutdinov, G. A. 1992, Preprint SAO RAS, No. 92

Gaposhkin, S. 1952, Harvard Obs. Ann., 115

Giridhar, S., Lambert, D., \& Gonzalez, G. 1998, PASP, 110, 671

Giridhar, S., Lambert, D., \& Gonzalez, G. 1999, PASP, 111, 1269

Giridhar, S., Lambert, D., \& Gonzalez, G. 2000, PASP, 112, 1559

Goswami, A., \& Prantzos, N. 2003, A\&A, 359, 191

Gratton, R. G., Caretta, E., Claudi, R., Lucatello, S., \& Barbieri, M. 2003, A\&A, 404,187
Johnson, J. 2002, ApJS, 139, 219

Jones, D. H. P., \& Fisher, J. L. 1984, A\&AS, 56, 449

Korotin, S. A., Andrievsky, S. M., \& Luck, R. E. 1999a, A\&A, 351, 168

Korotin, S. A., Andrievsky, S. M., \& Kostynchuk, L. Yu. 1999b, Ap\&SS, 260, 531

Korotin, S. A., \& Mishenina, T. V. 1999, ARep, 43, 533

Kovtyukh, V. V., \& Andrievsky, S. M. 1999, A\&A, 351, 597

Kovtyukh, V. V., Mishenina, T. V., Gorbaneva, T. I., et al. 2006, ARep, 50, 134

Kurucz, R. L. 1992, Rev. Mex. Astron. Astrofis., 23, 45

Leep, E. M., \& Wallerstein, G. 1981, MNRAS, 196, 543

Luck, R. E., \& Bond, H. E. 1985, ApJ, 292, 559

Preston, G., \& Wallerstein, G. 1963, ApJ, 138, 820

Samland, M. 1998, ApJ, 496, 155

Simmerer, J. A., Sneden, C., Cowan, J. J., et al. 2004, ApJ 617, 1091

Sobeck, J. S., Ivans, I. I., Simmerer, J. A., et al. 2006, AJ, 131, 2949

Stephens, A., \& Boesgaard, A. M. 2002, AJ, 123, 1647

Takeda, Y., Zhao, G., Takada-Hidai, M., et al. 2003, ChJAA, 3, 316

Zhang, H. W., \& Zhao, G. 2005, MNRAS, 364, 712 